\title{
Erroneous UTC Maintained by Timing Labs Due to Mix-Up Between Relativistic and Absolute Time
}

\author{
Gurcharn S. Sandhu ${ }^{1}$ \\ ${ }^{1}$ Independent Researcher, Punjab, India \\ Correspondence: Gurcharn S. Sandhu, K. No. 48, Sector-61, Mohali, Punjab 160062, India. Tel: 91-172-4657020. \\ E-mail: gurcharn.sandhu@gmail.com
}

Received: August 8, 2013 Accepted: September 24, 2013 Online Published: October 24, 2013

doi:10.5539/apr.v5n6p83

URL: http://dx.doi.org/10.5539/apr.v5n6p83

\begin{abstract}
In Relativity the Newtonian notions of absolute motion, absolute time, and absolute reference frame have been replaced with relative motion, relativistic time, and inertial reference frames in motion. Relativity is essentially rooted in the assumed isotropy of light speed in earth centered inertial (ECI) reference frame. Under the current procedures of satellite based time transfer, due to the assumed isotropy of light speed in ECI frame, an e-synchronous or relativistic time gets distributed to the master clocks of Timing laboratories located all over the globe. That is, the master clocks in all Timing Labs get e-synchronized instead of achieving absolute synchronization. It is erroneous to compute UTC from the weighted average of relativistic time maintained by these master clocks. The absolute synchronization mismatch between two e-synchronized clocks is given by the relation (D.U) $/ \mathrm{c}^{2}$, where $\mathbf{D}$ is the separation distance between the two clocks and $\mathbf{U}$ is the absolute velocity vector (unknown) of the earth. This absolute synchronization offset between the master clocks at two distant Timing Labs can be physically measured with an appropriate portable clock and such measurements can be used to determine the unknown absolute velocity $\mathbf{U}$ of earth. By incorporating the absolute velocity $\mathbf{U}$ in the time transfer software, we can account for the anisotropic speed of light in the ECI frame and thereby ensure the distribution of absolute time to different clocks all over the globe. In that case it should even be possible to achieve absolute synchronization in space clocks in deep space flights.
\end{abstract}

Keywords: relativistic, absolute, e-synchronization, time transfer, isotropic

\section{Introduction}

The International Atomic Time (TAI) and Coordinated Universal Time (UTC) are maintained at the International Bureau of Weights and Measures (BIPM) using data from some two hundred atomic clocks in over seventy national laboratories (Labs) spread over the globe. The clock comparisons that provide the data for the calculation of TAI are mainly carried out using GPS satellites. As per BIPM (2012) report, physical realizations of UTC $(\mathrm{k})$ are maintained in national metrology institutes or Labs contributing with their clock data to the BIPM. These Timing Labs strive their best to achieve sub-nanosecond accuracies in their UTC $(\mathrm{k})$ maintained with local clock ensembles. Yet, the $\operatorname{UTC}\left(\mathrm{k}_{1}\right)$ and $\mathrm{UTC}\left(\mathrm{k}_{2}\right)$ maintained by two different Timing Labs $\mathrm{k}_{1}$ and $\mathrm{k}_{2}$, separated by a long baseline D (say more than $1000 \mathrm{~km}$ ), could show a timing mismatch of hundreds of nanoseconds if ever compared with a portable precision atomic clock. While in the last century United States Naval Observatory (USNO) used to have an entire division that did nothing but portable clock calibrations world-wide, that division was abolished with the advent of satellite-based time transfer methods. However, at present most experts in the Time keeping field do not consider the physical verification of any timing mismatch between a pair of Timing Labs as feasible. That is because they believe that the time and frequency of all clocks gets influenced by any change in their position or velocity due to the Relativity effects. Such effects are taken for granted, since Relativity has apparently worked so well for more than hundred years now.

In historical perspective, the earth centered Ptolemaic model accurately explained the motions of heavenly bodies and was not seriously challenged for over 1300 years. The major strength of that model was its ability to satisfactorily explain all observations of heavenly bodies at that time. It could finally be discarded by Johannes Kepler and Galileo Galilei after more accurate instrumented observations contradicted the basic assumptions of that model. Similarly, the Relativity model developed by Albert Einstein in early 20th century could accurately explain all observations of physical phenomena that depended on light (or EM wave) signals. In major practical 
applications of the Relativity model, namely the GPS system and satellite based communication systems, the speed of light propagation is assumed to be isotropic in the earth centered inertial (ECI) reference frame. As such the Relativity model essentially implied a unique position for the ECI reference frame, in which the speed of light propagation was assumed to be an isotropic constant $\mathrm{c}$ under vacuum conditions. The generalized form of this assumption formed the second postulate of special theory of Relativity (SR) which effectively implies light speed isotropy in all inertial reference frames (IRF). The ECI frame is special in the sense that it is the only inertial reference frame, apart from Barycentric Celestial Reference Frame (BCRF), which is practically maintained by the International Earth Rotation and Reference Systems Service (IERS). These two reference frames, BCRF for our solar system and ECI for the earth-moon system are said to be the main IRF practically maintained by the IERS for use in our scientific applications. All other IRF in relative uniform motion are only hypothetical reference frames used for conducting thought experiments of SR and cannot be practically maintained by IERS for use in any scientific application.

The notions of isotropy of light speed in ECI frame, e-synchronization of clocks, relativistic time and Lorentz transformations constitute the SR model. On the other hand the anisotropy of light speed in ECI frame, absolute synchronization of clocks, absolute time and Galilean transformations constitute a set of notions under the Newtonian model of absolute space and time. In section 2 we examine the Relativity model which has survived mainly for want of a better alternative. If the speed of light is not an isotropic constant in ECI frame then it must be isotropic in a bigger, universal frame. However, all attempts of scientific community to establish such an absolute or universal frame, by detecting the absolute motion of earth in that frame, have failed so far. Therefore, all attempts to invalidate the Relativity model are, in essence, synonymous to the detection of absolute motion of earth and thereby establishing an absolute reference frame in which the speed of light is an isotropic constant. In section 3 we mainly distinguish between the notions of relativistic and absolute time while in section 4 we distinguish between the absolute and e-synchronization conventions. Finally in sections 5 and 6 we examine the UTC maintained by various Timing Labs.

\section{Postulates and Implicit Assumptions of Relativity}

The first postulate of SR enunciates the special "Principle of Relativity" in which it has been assumed that the laws of physics are the same in all inertial reference frames, regardless of their state of motion. Physically, this means that all IRF in relative uniform motion are equivalent for the description of the laws of Nature and that there is no absolute or preferred frame of reference. The second postulate enunciates the constancy and isotropy of the speed of light in all IRF.

\subsection{Logical Flaw in Special Principle of Relativity}

The special Principle of Relativity implies that all IRF in relative motion are equivalent and that no particular IRF can be treated as a preferred frame which could be physically distinguished from other IRF. Basically, all laws of Nature remain valid in observable universe, independent of reference frames. The laws of Nature and characteristic interactions among material particles cannot be influenced by the arbitrarily defined coordinate systems. However, in physics we quantify the laws of Nature, so as to represent them through certain mathematical equations involving various dimensional physical parameters. The form of mathematical representation of the laws of Nature can change with the change in reference frame but not the laws themselves. It is wrong to assume any linkage or correlation between the laws of Nature and the arbitrarily defined inertial reference frames (Sandhu, 2011b). Hence, there is a logical flaw in the principle of Relativity since it implies a correlation between the laws of physics and the arbitrarily defined reference frames. Fundamentally the laws of Nature and characteristic interactions among material particles and fields cannot be influenced by or depend upon the arbitrarily defined coordinate systems. Therefore, it is wrong to infer the equivalence of all IRF on the pretext of laws of Nature.

Let the BCRF of our solar system represent an IRF K, and let K' be any other IRF moving in uniform translation relative to BCRF. We know that in BCRF, being a center of mass (CoM) reference frame, the total linear momentum of all particles within the solar system will be zero, but not in any other IRF K' which is in motion relative to BCRF. Further, the total kinetic energy of all particles within the solar system will be a minimum in BCRF, and not in any other IRF K'. Even though the magnitude of total kinetic energy and total momentum of the solar system will be different in different IRF K', it will be a unique minimum kinetic energy and zero momentum only in the CoM reference frame K'. This fundamental difference between their total momentum and total kinetic energies, becomes a distinguishing feature between IRF $\mathrm{K}$ and $\mathrm{K}$ ' and sets the reference frame $\mathrm{K}$ or the CoM reference frame as the unique, preferred reference frame for our solar system.

Further, let us consider an inertial reference frame K' moving with relative uniform velocity of $0.9 \mathrm{c}$ with respect 
to BCRF. In BCRF, all planetary orbits around the sun are quite stable. At high speeds the dynamic mass, representing total energy content, of a body increases significantly. From the K' frame, the dynamic mass of the sun and planets will be "seen" to be more than double due to the Lorentz gamma factor. With length contraction only in the direction of relative motion, the planetary orbits as computed from K' will tend to shrink and become unstable. This drastic difference between the nature of planetary orbits as computed from BCRF and K' shatters the supposed equivalence of all IRF in relative motion.

\subsection{Logical Flaw in the Second Postulate of SR}

The second postulate of SR depicts an assumption that the speed of light propagation under vacuum conditions is the same isotropic constant $\mathrm{c}$ in all IRF such as the ECI frame. It is well known from Maxwell's theory that the speed of light propagation, under vacuum conditions, depends on the permittivity $\varepsilon_{0}$ and permeability $\mu_{0}$ of the physical space. Since permittivity and permeability are properties of the physical space, the speed of light in vacuum is also a property of physical space and cannot be derived from the metric properties of coordinate space (Sandhu, 2009). For example, in the case of a homogeneous and isotropic material medium, when the speed of sound or pressure waves depend on the physical properties of that medium, that speed of such wave propagation is always defined with respect to a reference frame at rest in that medium. In the same way, when the speed of light wave propagation in physical space depend on its physical properties, that speed of light wave propagation is always defined with respect to a reference frame at rest or fixed in physical space. Hence the speed c of light propagation in physical space can be an isotropic constant only with respect to an absolute reference frame. Therefore, it is wrong to assume light speed isotropy in all IRF in relative motion.

To comply with the assumption of light speed isotropy in all IRF, the notion of time as an absolute measure of change has been sacrificed in SR, leading to the notions of relativistic time and consequent length contractions. According to SR, the time interval dt of a standard atomic clock and a length segment dx of a standard meter rod, will be seen to be different in each of the infinitely many IRF in relative motion. However, the notions of length contraction and time dilation are not physical but hypothetical remote observation effects, resulting from measurements made by fictitious observers from abstract IRF in relative motion. A rod of length $\mathrm{L}_{0}$ at rest in BCRF will be found to be of shorter length L when hypothetically measured in an IRF K' moving parallel to the length of the rod. We say "hypothetically measured" because as per the prescribed method of measurement, the measuring rods are supposed to be carried in the moving IRF K' while the rod to be measured is located in the $\mathrm{BCRF}$, and the measurements are to be carried out through exchange of light signals. Practically it is impossible to carry out such measurements.

Further, to elaborate the fictitious nature of length contraction, consider a thin spherical glass shell which is sure to break if compressed or deformed from outside. As per SR, when such a glass shell is viewed from a moving IRF, the diameter of glass shell is supposed to get contracted in the direction of relative motion but the shell will not physically break. That is because the shell cannot undergo any physical change just because of its being viewed by observers in motion. This confirms that not only the notion of length contraction is fictitious but the basic assumption of light speed isotropy, in all IRF in relative motion, is also wrong.

\subsection{Logical Flaw in the Concept of Curved Spacetime}

Relativity has extended the notion of three dimensional (3D) coordinate space to 4D spacetime to facilitate geometrical representations of gravitational trajectories. The invariance of a spacetime interval with respect to Lorentz transformations is fundamentally dependent on the assumption of light speed isotropy in all IRF. As such the use of a 4D spacetime continuum as a geometric background, with invariance of spacetime interval, is simply a mathematical abstraction representing the light speed isotropy in all IRF. Such abstraction is justified as long as we are dealing with mathematical models of a physical situation, such as the assumed isotropy of light speed in all IRF. But in the geometric interpretation of Gravitation, where matter physically influences the metric and hence the curvature of spacetime, the 4D spacetime continuum is implied to be a physical entity. Fundamentally, the matter as a physical entity cannot influence an abstract geometric construct.

If we don't assume the spacetime continuum to be a physical entity, then the General theory of Relativity (GR) can no longer provide a theory of gravitation. In that case the 4D curved spacetime of GR just represents an abstract geometric construct, with differential scale four dimensional coordinate system used to model the gravitational trajectories of particles as geodesic curves. That is, without assuming the spacetime continuum to be a physical entity, GR reduces to an abstract mathematical model for computing gravitational trajectories and associated analytical studies. But to elevate this mathematical model to the status of a theory of gravitation, providing foundational basis for the phenomenon of gravitation, Einstein had to assume the spacetime continuum to be a physical entity which could even be deformed and curved. Albert Einstein had asserted in a matter of fact 
way (Einstein, 1916), "the world in which we live is a four-dimensional spacetime continuum." Broadly GR implies that, "mass curves spacetime, and spacetime tells the mass how to move." The spacetime continuum of GR is not a Euclidean Continuum with uniform scale coordinate system. The Riemannian 3D space of GR is defined to be a deformable space which is generally perceived as curved space. However, Sandhu (2011a) has shown that the 4D spacetime of GR is not a physical entity but just an abstract mathematical construct with differential scale coordinate system.

\section{Time and Its Measurement}

Time is what we measure with clocks - all sorts of clocks. All natural phenomena in the Universe are intrinsically dynamic with a dominant feature of continuous change. Observable changes may be random, monotonic or cyclic. For cyclic changes, suppose when parameter "a" goes through $\mathrm{N}_{\mathrm{a}}$ cycles of change, parameter " $d$ " goes through $\mathrm{N}_{\mathrm{d}}$ cycles. We can then compare the two sets of changes by saying that while parameter "a" completes one cycle, parameter " $\mathrm{d}$ " completes $\left(\mathrm{N}_{\mathrm{d}} / \mathrm{N}_{\mathrm{a}}\right)$ cycles. And this comparison between different sets of changes gives rise to the notion of comparatively slow or fast changes and hence to the notion of time as a measure of rate of change (Sandhu, 2009). In Nature, there are a large number of physical processes, which undergo cyclic changes. Depending on the consistency of such cyclic changes and the convenience of their measurement, we may select any one of them as our reference scale for relative measurement of change or the reference scale for time. The angular position of a planet in orbit around the Sun, the rotational motion of earth, the position of a pendulum oscillating about a mean, the vibrations of many mechanical and electro-magnetic systems are all examples of physical processes that undergo cyclic changes. Any such system or process could be adopted as a reference scale for relative measurement of change or measurement of Time.

\subsection{The Clock}

However, the most important aspect of the measure of time is that any specific time interval "dt" between two sequential events consists of the elapsed number of cyclic oscillation periods of the reference time scale adopted. A clock is the device that counts or sums up all such elapsed number of oscillation periods of the reference time scale. Modern precision clocks are characterized by their highly consistent electronic oscillations or frequency and a precision counter that sums up such oscillation cycles from some specified zero reference. Two precision clocks can be regarded as identical if their characteristic frequencies match exactly and are expected to remain matched over their service life. Modern precision atomic clocks measure any specific time interval "dt" by summing up or counting their characteristic electronic oscillations during that interval.

A given clock can be simultaneously regarded as located in the Earth Centered Earth Fixed (ECEF) reference frame, ECI frame, BCRF and the Universal reference frame. Any such clock has no in-built mechanism or sensor to automatically sense its position or velocity in any of the arbitrarily defined coordinate reference frames. As such, no clock can sense its change in position or velocity in any of the arbitrarily defined coordinate reference frames. Hence, the frequency and displayed time of all precision atomic clocks, located within the ECI frame, are completely independent of their position and velocity in the ECI frame. Further, the precision timing readings and comparisons of modern atomic clocks are digitally recorded on co-located computers and then transferred or distributed to different users or observers through telecommunication links. It is during this time transfer or distribution through telecommunication links that we make use of the assumption that the speed of light (or EM waves) is an isotropic constant in the ECI reference frame under vacuum conditions.

\subsection{Absolute and Relativistic Time}

Since the frequency and displayed time of all precision atomic clocks, located within the ECI or any other IRF, are completely independent of their position and velocity in that frame, the time measured by these physical or real clocks is the Newtonian absolute time. That is, the absolute time as measured by all precision atomic clocks is completely independent of their position or velocity in any reference frame. On the other hand, Albert Einstein introduced a different notion of time in his 1905 paper (Einstein, 1905), “...We have not defined a common 'time' for $A$ and $B$, for the latter cannot be defined at all unless we establish by definition that the 'time' required by light to travel from A to B equals the 'time' it requires to travel from $B$ to A". The "common time" thus defined in SR is not the absolute but relativistic time and this definition formed the basis for Einstein synchronization convention or simply the "e-synchronization" of clocks. This arbitrary definition of "common" or relativistic time constitutes a fundamental departure from the Newtonian notion of absolute time. Of course, this departure was required to support the assumed isotropy of light speed c in all IRF. When the clocks at two spatially separated points $\mathrm{A}$ and $\mathrm{B}$ are adjusted or set to the relativistic time, it will ensure that the time interval measured for the light to travel from A to B will be equal to the time interval measured for the light to travel from B to A. In other words, if a group of identical clocks are adjusted or set to the relativistic time then the light speed 
measured with such clocks will always be isotropic. Reversing the argument, if precision time of a standard clock is distributed to a group of identical clocks through light (or EM) signals by assuming light speed isotropy in the chosen reference frame, then the time thus transferred to these clocks will be relativistic time and not the absolute time.

The distinction between absolute time and relativistic time is often missed. Whenever the word "time" occurs in discussions or analysis, the opponents of Relativity always take it as the Newtonian "absolute time" because physical clocks inherently measure absolute time, but the supporters of Relativity always take it as the relativistic time because the notion of absolute time does not exist in Relativity. The notion of "time" used in Lorentz transformations is always the relativistic time. Since the relativistic time and e-synchronization of two precision clocks will change with change in their state of motion, most Relativity supporters have mistakenly come to believe that the inherent time displayed by the precision clocks (i.e. the absolute time) will also change with change in their state of motion. However, the underlying reason behind this mistaken belief is that the notion of "clock" used in all thought experiments of Relativity, is not that of a physical clock but that of a hypothetical clock which automatically adjusts itself to relativistic time with change in its position or velocity. Thus the erroneous belief that the inherent time displayed by physical clocks will change with change in their state of motion with respect to an observer, is based on the mix-up between the notions of relativistic and absolute time.

None of the physical clocks have any built-in sensor to detect its speed with respect to an arbitrarily defined IRF, like ECI or BCRF. Not to speak of a precision clock, even the 21 st century sophisticated cutting edge technology is unable to develop any sensor for fitment in a spaceship, which could measure the speed of the spaceship in deep space, with respect to an arbitrarily defined IRF like ECI or BCRF. Even if some clocks are somehow blessed with a "sixth sense" and know their speed with respect to an arbitrarily defined IRF, then such clocks must include a sort of feed-back control mechanism in their basic design for adjusting their frequency based on the feed-back parameter of speed. But such a feedback control mechanism is never incorporated in the design of any physical clock. As such any physical clock cannot adjust its frequency or phase, proportional to its speed with respect to an arbitrary IRF like ECI or BCRF.

\section{Synchronization}

When the frequency and phase or displayed time of two or more precision atomic clocks are matched or adjusted to match exactly in close-by position, the clocks are said to be in absolute synchronization. Since the time and frequency of any precision atomic clock is completely independent of their state of motion or velocity in any chosen reference frame, the absolute synchronization of two or more clocks will not be disturbed by any change in their state of motion. Therefore, when two or more precision atomic clocks are in absolute synchronization, they will display absolute time, regardless of their state of motion with respect to any observer. However, according to the arbitrary definition of "common time", when the displayed time of two spatially separated precision atomic clocks is adjusted to ensure that the to and fro propagation time of light signal between them will be measured to be the same, the two clocks are said to be synchronized under Einstein convention or simply e-synchronized.

The word "synchronization" is often used in two different connotations. The supporters of Relativity always use the word "synchronization" to imply e-synchronization of two or more clocks whereas the opponents of Relativity always use the word "synchronization" to imply absolute synchronization of two or more clocks. When two or more identical precision clocks are synchronized in close-by position, they will retain their absolute synchronization even when they are moved to different locations or moved at different relative velocities. That is, just as the "time and frequency" of individual precision clocks is independent of their state of motion, the absolute synchronization of two or more identical precision clocks is also independent of the state of their motion. However, when two or more identical precision clocks are once e-synchronized in one position, their e-synchronization will not be retained when they are moved to different locations or moved at different relative velocities. That is, the relativistic time displayed by two or more e-synchronized clocks will not remain independent of their state of motion.

\subsection{E-Synchronization Process}

Since the speed of light in vacuum is a property of physical space, it can be an isotropic constant only with respect to an absolute reference frame. Consider a line segment $\mathrm{AB}$ of length $\mathrm{D}$, moving in the absolute reference frame at velocity $U_{a b}$ along $A B$. The time taken by a light pulse to propagate from point $A$ to $B\left(T_{a b}\right)$, for $U_{a b}<<c$ (in which case powers of $\mathrm{U}_{\mathrm{ab}} / \mathrm{c}$ can be neglected), is

$$
\mathrm{T}_{\mathrm{ab}}=\mathrm{D} /\left(\mathrm{c}-\mathrm{U}_{\mathrm{ab}}\right)=\mathrm{D} / \mathrm{c}+\mathrm{D} \cdot \mathrm{U}_{\mathrm{ab}} / \mathrm{c}^{2} .
$$


Similarly, if $\mathrm{T}_{\mathrm{ba}}$ is the time taken by a pulse of light to propagate from point $\mathrm{B}$ to $\mathrm{A}$, then,

$$
\mathrm{T}_{\mathrm{ba}}=\mathrm{D} /\left(\mathrm{c}+\mathrm{U}_{\mathrm{ab}}\right)=\mathrm{D} / \mathrm{c}-\mathrm{D} \cdot \mathrm{U}_{\mathrm{ab}} / \mathrm{c}^{2}
$$

From Equations (1) and (2), it can be shown that,

$$
\mathrm{U}_{\mathrm{ab}} / \mathrm{c}=\left(\mathrm{T}_{\mathrm{ab}}-\mathrm{T}_{\mathrm{ba}}\right) /\left(\mathrm{T}_{\mathrm{ab}}+\mathrm{T}_{\mathrm{ba}}\right)
$$

When a line segment $A B$ is moving with absolute velocity $U_{a b}$ along $A B$, a signal pulse will take longer to propagate from the trailing end (A) to the leading end (B) but will take shorter time from B to A. Equation (3) shows that the absolute velocity $\mathrm{U}_{\mathrm{ab}}$ can be measured by measuring the to and fro pulse propagation times between points A and B. Sandhu (2010) has established the feasibility of a doable experiment for detection of absolute motion on this basis. To meet the requirements of Einstein's arbitrary definition of common time, the clocks A and B will have to be adjusted or "synchronized" in such a way that the leading end clock (B) is set to lag behind the trailing end clock by D. $U_{a b} / c^{2}$. That is, when the instantaneous time reading on clock $A$ is $t_{a}$ the corresponding reading on clock $\mathrm{B}$ will be,

$$
\mathrm{t}_{\mathrm{b}}=\left(\mathrm{t}_{\mathrm{a}}-\mathrm{D} \cdot \mathrm{U}_{\mathrm{ab}} / \mathrm{c}^{2}\right)
$$

When a light pulse emitted from $A$ at an instant $t_{a}$, reaches $B$ in time $T_{a b}$, the clock time at $B\left(T_{b}\right)$ will be,

$$
\begin{aligned}
\mathrm{T}_{\mathrm{b}} & =\mathrm{t}_{\mathrm{b}}+\mathrm{T}_{\mathrm{ab}}=\left(\mathrm{t}_{\mathrm{a}}-\mathrm{D} \cdot \mathrm{U}_{\mathrm{ab}} / \mathrm{c}^{2}\right)+\mathrm{T}_{\mathrm{ab}} \\
& =\left(\mathrm{t}_{\mathrm{a}}-\mathrm{D} \cdot \mathrm{U}_{\mathrm{ab}} / \mathrm{c}^{2}\right)+\mathrm{D} / \mathrm{c}+\mathrm{D} \cdot \mathrm{U}_{\mathrm{ab}} / \mathrm{c}^{2}=\mathrm{t}_{\mathrm{a}}+\mathrm{D} / \mathrm{c}
\end{aligned}
$$

With such an adjustment or "e-synchronization" of clocks, the measured pulse propagation time from A to B $\left(T_{b}-t_{a}\right)$ will become $D / c$. Similarly, when the reading on clock A is $t_{a}$ and the corresponding reading on clock B is $t_{b}=\left(t_{a}-D \cdot U_{a b} / c^{2}\right)$, let a pulse of light be emitted from B towards A. The pulse will reach A in time $T_{b a}$ (Equation 2) when the clock reading on clock $A$ is $T_{a}$ '.

$$
\begin{aligned}
\mathrm{T}_{\mathrm{a}}{ }^{\prime} & =\mathrm{t}_{\mathrm{a}}+\mathrm{T}_{\mathrm{ba}}=\mathrm{t}_{\mathrm{a}}+\mathrm{D} / \mathrm{c}-\mathrm{D} \cdot \mathrm{U}_{\mathrm{ab}} / \mathrm{c}^{2} \\
& =\left(\mathrm{t}_{\mathrm{a}}-\mathrm{D} \cdot \mathrm{U}_{\mathrm{ab}} / \mathrm{c}^{2}\right)+\mathrm{D} / \mathrm{c}=\mathrm{t}_{\mathrm{b}}+\mathrm{D} / \mathrm{c}
\end{aligned}
$$

The measured pulse propagation time from $B$ to $A\left(T_{a}{ }^{\prime}-t_{b}\right)$ will therefore become $D / c$. This shows that with such an adjustment or e-synchronization of the two clocks, the measured pulse propagation time from $\mathrm{A}$ to $\mathrm{B}$ will always be equal to the measured pulse propagation time from B to A. This is the main characteristic of e-synchronization in SR. In contrast, two identical clocks synchronized in close-by position will retain their absolute synchronization. But when the clocks, separated by distance D, are e-synchronized through time transfer by assuming an isotropic light speed, the clocks get synchronized for relativistic time in which the leading end clock (B) gets set to lag behind the trailing end clock by D. $U_{a b} / \mathrm{c}^{2}$.

\subsection{Lorentz Transformation for Time}

Let us consider an inertial reference frame in which the speed of light is known to be an isotropic constant under vacuum conditions and designate it as fixed frame $\mathrm{K}$. Let an observer $\mathrm{O}$ use a Cartesian coordinate system (X, Y, $\mathrm{Z}$ ) fixed in frame $\mathrm{K}$. Let $\mathrm{t}$ denote the instantaneous time on all clocks in frame $\mathrm{K}$ that are in absolute synchronization. Let a line segment $A B$, aligned along $X$-axis, move in frame $K$ with a velocity $U_{a b}=v$, along $\mathrm{AB}$ or positive $\mathrm{X}$-axis. Let another observer $\mathrm{O}$ ' moving with the line segment $\mathrm{AB}$, use an inertial reference frame $\mathrm{K}^{\prime}$ with a Cartesian coordinate system $\left(X^{\prime}, Y^{\prime}, Z^{\prime}\right)$ such that $X$ and $X^{\prime}$ axes are collinear. Let the origin of the two coordinate systems $(X, Y, Z)$ and $\left(X^{\prime}, Y^{\prime}, Z^{\prime}\right)$ coincide at an instant when $t_{0}=t^{\prime}{ }_{0}=0$. At another instant of time $t$, let the $\mathrm{x}$ coordinate of point $\mathrm{A}$ be $\mathrm{x}_{\mathrm{a}}$ and that of point $\mathrm{B}$ be $\mathrm{x}_{\mathrm{b}}$ such that $\mathrm{x}_{\mathrm{b}}-\mathrm{x}_{\mathrm{a}}=\mathrm{AB}=\mathrm{D}$. In this so called standard configuration, Lorentz transformation for relativistic time $t^{\prime}$ in moving frame $\mathrm{K}$ ' for points $\mathrm{A}$ and $\mathrm{B}$, for $\mathrm{v}<<\mathrm{c}$ (in which case powers of $\mathrm{v} / \mathrm{c}$ can be neglected), will be given by,

$$
\begin{aligned}
& \mathrm{t}_{\mathrm{a}}{ }^{\prime}=\mathrm{t}-\mathrm{x}_{\mathrm{a} \cdot} \cdot \mathrm{v} / \mathrm{c}^{2} \\
& \mathrm{t}_{\mathrm{b}}{ }^{\prime}=\mathrm{t}-\mathrm{x}_{\mathrm{b}} \cdot \mathrm{v} / \mathrm{c}^{2}
\end{aligned}
$$

That is, when the instantaneous time on clocks in fixed reference frame $\mathrm{K}$ is $\mathrm{t}$, the corresponding time for the clocks located on the line segment $\mathrm{AB}$ in the moving frame $\mathrm{K}$ ' is given by Lorentz transformation for time, as a function of their instantaneous location $x$ and velocity v. Subtracting Equation (7) from (8), the difference in relativistic time $\mathrm{t}^{\prime}$ between the clocks $\mathrm{A}$ and $\mathrm{B}$ is given by,

Or,

$$
\begin{gathered}
\mathrm{t}_{\mathrm{b}}{ }^{\prime}-\mathrm{t}_{\mathrm{a}}{ }^{\prime}=-\left(\mathrm{x}_{\mathrm{b}}-\mathrm{x}_{\mathrm{a}}\right) \cdot \mathrm{v} / \mathrm{c}^{2}=- \text { D.v } / \mathrm{c}^{2} \\
\mathrm{t}_{\mathrm{b}}{ }^{\prime}=\mathrm{t}_{\mathrm{a}}{ }^{\prime}-\text { D.v } / \mathrm{c}^{2}
\end{gathered}
$$

This result is identical to the one given by Equation (4), which implies that when a line segment $\mathrm{AB}$ of length $\mathrm{D}$ is in motion at velocity $\mathrm{v}$ along $\mathrm{AB}$, the leading end clock $\mathrm{B}$ lags in time reading by $\mathrm{D} . \mathrm{v} / \mathrm{c}^{2}$ with respect to the 
trailing end clock A. As shown above, this is the requirement of e-synchronization of clocks to ensure that the measured pulse propagation time from $\mathrm{A}$ to $\mathrm{B}$ will always be equal to the measured pulse propagation time from $\mathrm{B}$ to A. Therefore, the Lorentz transformation provides a convenient method of computing the relativistic time for clocks in a moving reference frame K'. When all clocks in a moving reference frame K' are somehow set to or adjusted to the relativistic time obtained from Lorentz transformation for time, the speed of light measured with such clocks will always be found to be isotropic in that frame.

However, there is a major ambiguity in actually setting or adjusting the clocks in frame K' to the relativistic time given by Lorentz transformation. As seen above, physical clocks which inherently measure absolute time, cannot automatically adjust themselves to display relativistic time when in motion. Since some supporters of Relativity believe that all clocks in moving reference frame K' automatically get set to the relativistic time given by Lorentz transformation, it implies that the clocks used in thought experiments of Relativity are hypothetical clocks. On the other hand, some supporters of Relativity do believe that physical clocks do not experience any adjustment or change in their instantaneous time readings in any reference frame. It is only an observational effect of Relativity that an observer in fixed frame $\mathrm{K}$ will somehow read or observe the relativistic time on the clocks in moving frame $\mathrm{K}^{\prime}$ in accordance with the predictions of Lorentz transformation. In reality, however, there is neither any automatic adjustment of clock readings to relativistic time nor any magical observational effect in which instantaneous clock readings are seen to be relativistic by an observer in relative motion. Lorentz transformation merely implies that if the instantaneous time readings of clocks in a moving reference frame K' are somehow set to the relativistic values given by the transformation, then the speed of light measured with these clocks in frame K' will be found to be isotropic.

Practically, of course, there is just one method of setting or adjusting the instantaneous time readings of clocks in moving frame $\mathrm{K}$ ' to the relativistic time values. That is by distributing the instantaneous time of a reference clock to all other clocks in frame K' by assuming the isotropy of light speed in the moving frame K'. Obviously, when we transfer the instantaneous time of a reference clock to any other clock in the moving frame through light (or EM) signals on the assumption of isotropy of light speed, the light speed measured with such clocks, under vacuum conditions, will always be found to be isotropic. Hence the time transferred to the clocks in moving frame $\mathrm{K}^{\prime}$, on the assumption of light speed isotropy in $\mathrm{K}$ ', will always be e-synchronous or relativistic. If the vacuum speed of light is measured with absolute synchronized clocks using absolute time, it will be found to be anisotropic in the ECI frame. Of course, Relativity does not use absolute time or absolute synchronization of precision clocks; that is why the anisotropy of light speed in the ECI frame cannot be detected or measured by using the Relativity model. However, the most important distinguishing feature here is that the physical precision atomic clocks inherently measure the absolute time and not the relativistic time.

\section{Time Transfer through Satellite Communication}

Sandhu (2012) has shown that when two clocks A and B are synchronized through a GPS satellite in common view mode, by assuming an isotropic speed of light propagations in ECI frame, their synchronization is equivalent to e-synchronization. Therefore, when we synchronize two or more clocks through satellite communication, by assuming light speed isotropy in ECI frame, they get e-synchronized to display relativistic time. In contrast, two identical clocks synchronized in close-by position attain absolute synchronization and display absolute time. In essence, the time distributed through satellite links, on the assumption of isotropy of light speed in the ECI frame, is the relativistic time and not the absolute time. Inherently all clocks measure the absolute time, until and unless specifically adjusted or e-synchronized to display relativistic time.

Consider two national Timing Labs which are regularly conducting clock comparisons through GPS satellites and designate them as Lab A and Lab B. Let the separation distance between the two Labs, with calibrated position coordinates, be $\mathrm{D}$ and let $\mathbf{U}$ be the absolute velocity vector (unknown) of the earth. Let the UTC time maintained at the two Labs, with their primary standard Cesium atomic clocks, be $\mathrm{T}_{\mathrm{UTC}}(\mathrm{A})$ and $\mathrm{T}_{\mathrm{UTC}}(\mathrm{B})$. In the normal process of conducting clock comparisons through GPS satellites in common view mode, Labs record their measurement data in CGGTTS format. The CGGTTS data format (Weiss \& Thomas, 1994) at the recording Labs contains information regarding satellite number (PRN) and the satellite track time (STTIME). After accounting for the estimated signal propagation delays and the propagation time for the known distance between the satellite and the receiver (with assumed isotropy of light speed), normal time difference (REFGPS) between the laboratory reference clock $\left(\mathrm{T}_{\mathrm{UTC}}\right)$ and the satellite GPS time $\left(\mathrm{T}_{\mathrm{GPS}}\right)$ is recorded as,

$$
\begin{aligned}
& \operatorname{REFGPS}(\mathrm{A})=\mathrm{T}_{\mathrm{UTC}}(\mathrm{A})-\mathrm{T}_{\mathrm{GPS}} \\
& \operatorname{REFGPS}(\mathrm{B})=\mathrm{T}_{\mathrm{UTC}}(\mathrm{B})-\mathrm{T}_{\mathrm{GPS}}
\end{aligned}
$$

The recorded timing data in CGGTTS format can be periodically exchanged between the recording Labs to 
compute the normal synchronization offset for a common PRN and STTIME as,

$$
\operatorname{REFGPS}(\mathrm{A})-\operatorname{REFGPS}(\mathrm{B})=\mathrm{T}_{\mathrm{UTC}}(\mathrm{A})-\mathrm{T}_{\mathrm{UTC}}(\mathrm{B})
$$

A zero value of this synchronization offset will imply e-synchronization between the master clocks at $A$ and $B$ Labs. But when the clocks are e-synchronized by assuming an isotropic light speed, the clocks get synchronized for relativistic time in which the leading end clock gets set to lag behind the trailing end clock by $\mathbf{D} . \mathbf{U} / \mathrm{c}^{2}$. The absolute synchronization offset between the master clocks at two distant timing Labs can be physically measured with an appropriate portable clock and such measurements can in fact be used to determine the unknown absolute velocity vector $\mathbf{U}$ of earth. On this basis a simple doable experiment has been proposed in a recent paper (Sandhu, 2012) titled, "Detection of absolute motion through measurement of synchronization offsets". By incorporating the absolute velocity vector $\mathbf{U}$ in the time transfer software, we can account for the anisotropic speed of light in the ECI reference frame and thereby ensure the distribution of absolute time to different clocks all over the globe.

Since the reference clock time is distributed to GPS satellites through telecom links by assuming the isotropy of light speed in the ECI frame, the GPS system time thus distributed is also a relativistic time and not the absolute time. The OPERA Collaboration (2011) measured the neutrino velocity over a known baseline of about $730 \mathrm{~km}$. At CERN, the accelerator chain was time stamped with UTC time obtained from GPS receiver XL-DC, whereas a GPS receiver ESAT-2000 provided the UTC time at LNGS. In July 2007, the synchronization mismatch between CERN and LNGS clocks, measured with a portable atomic clock, was found to be about $424 \mathrm{~ns}$. In 2008, two new PolaRx2e GPS receivers, with Cesium clocks, were installed as additional systems at CERN and LNGS to correct the purported erratic behavior of the two old GPS systems. The PolaRx2e receivers are geodetic time receivers with an accuracy of one ns. In the LNGS and CERN timing system, the PolaRx2e receivers were used to correlate the CERN system time $T_{\text {UTC }}(C)$ with the OPERA time $T_{\text {UTC }}(G)$. The difference between $T_{\text {UTC }}(C)$ and $\mathrm{T}_{\mathrm{UTC}}(\mathrm{G})$ represented the synchronization mismatch between the system times of CERN and LNGS, each of which was independently synchronized to the common GPS system time. However, as shown at figure 9.1 of the $\mathrm{PhD}$ Thesis of Brunetti (2011), the OPERA event times are being corrected for the synchronization mismatch of the order of $240 \mathrm{~ns}$ between CERN and LNGS. Such an high order of synchronization mismatch between the UTC times at CERN and LNGS, with diurnal fluctuations of about $60 \mathrm{~ns}$, presented clear evidence of an absolute synchronization mismatch between the two GPS receivers located at the ends of a long baseline.

\section{UTC Maintained by Global Timing Labs}

The International Atomic Time (TAI) and UTC are maintained at BIPM by using weighted averages of clock comparison data from some two hundred atomic clocks in over seventy national metrology institutes or Timing Labs. Many different methods for clock comparisons are currently in operation, including GPS common view, two-way satellite time and frequency transfer (TWSTFT) and some combined time links. The clock comparisons that provide the data for the calculation of TAI and UTC are mainly carried out using GPS and TWSTFT. Physical realizations of UTC $(\mathrm{k})$ are maintained in national Timing Labs contributing with their clock data to the BIPM. In the process of maintaining UTC (k), each Lab records clock offsets between UTC $(k)$ and the GPS time in CGGTTS file format as REFGPS(k), and sends about 90 such records to BIPM every day. The difference between simultaneous measurements (common STTIME) from two locations (Labs A and B), with a common view satellite (common PRN), determine the clock offsets between two participating Labs as, [REFGPS(A)REFGPS(B)]. Near zero value of such clock offsets implies a synchronous matching of the master clock time (or phase) at the two Labs.

However, the ambiguity in the notion of "synchronous matching", whether it means absolute synchronization or e-synchronization, becomes a source of error in the current methods of time transfer. The time distributed to the global set of Timing Labs through satellite communication, with the assumed isotropy of light speed in ECI frame, is relativistic time and not absolute time. By minimizing the clock offsets [REFGPS(A)-REFGPS(B)] between two participating Labs A and B, to the inherent noise level of the distribution system, only the time (or phase) of the master clocks gets adjusted to the relativistic time without affecting the inherent frequency of these clocks. Currently, the atomic frequency standards have achieved unprecedented stability and accuracy, and further advances in this field are not hampered by the distribution of relativistic time to the global Timing Labs. The ensemble of primary frequency standards are statistically consistent and the frequency uncertainty of TAI is approximately $3 \times 10^{-16}$. Therefore, in spite of the current distribution of relativistic time to the global Timing Labs under assumed isotropy of light speed in ECI frame, the stability and accuracy of primary and secondary frequency standards continues to be improved steadily. During the past decade very significant advances have been made in the field of optical frequency metrology, concerning both ultra-stable optical frequency standards 
as well as the means to compare frequencies locally using the optical comb technique. The stabilities of time and frequency transfer techniques currently and routinely used for clock comparisons around the world, are insufficient for comparisons between the best optical frequency standards (CCTF-19, 2012).

Even though the frequency of various primary frequency standards is highly stable and accurate, the relativistic time distributed to and maintained at the master clocks of global Timing Labs, UTC(k), cannot be said to be equally stable and accurate. Due to existing mix up between the notions of relativistic and absolute time, the relativistic time maintained at the global Timing Labs is normally referred as "time" and de-facto treated as absolute time. Therefore, the inherent difference between the relativistic and absolute times manifests itself as the inaccuracy or error in UTC $(\mathrm{k})$ maintained at the global Timing Labs. This error can be easily verified by physically comparing the $\mathrm{UTC}\left(\mathrm{k}_{1}\right)$ and $\mathrm{UTC}\left(\mathrm{k}_{2}\right)$ of two distant Timing Labs with a portable precision atomic clock, which is likely to show a mismatch of the order of a few hundred nanoseconds.

However, the error in UTC $(\mathrm{k})$ maintained at various Timing Labs is much more deep rooted than the simple ambiguity between the notions of relativistic and absolute time. We had to assume the isotropy of speed of light in ECI frame for want of any alternative. Since the Relativity model had been developed on the assumption of isotropy of light speed in all IRF, perforce we had to adopt the Relativity model, with all associated implications, wherever we had to assume the isotropy of light speed. By adopting the Relativity model, we had to discard the notions of absolute time and absolute synchronization in favor of relativistic time and e-synchronization. Further, implicitly we applied the notions of hypothetical clocks used in thought experiments of Relativity to the physical precision atomic clocks developed much after the advent of Relativity model. As such some supporters of Relativity tend to believe that due to relativistic effects, the time and frequency of real physical clocks also varies with change of their state of motion just as the time and frequency of hypothetical clocks varies in thought experiments of Relativity. Basically the time and frequency of a real physical portable atomic clock are independent of its position and velocity and hence the measurements made with a portable clock are valid and true. Hence any discrepancy in UTC(k) maintained at various Timing Labs, measured with a portable clock can be attributed to the anisotropy of light speed in ECI frame.

\section{Conclusion}

Relativity model is essentially founded on the light speed isotropy in all inertial reference frames and specifically in the ECI frame. Modern precision atomic clocks are characterized by their highly consistent electronic oscillations or frequency and a precision counter sums up such oscillation cycles to measure time. Since no physical clock can sense its change in position or velocity in any coordinate reference frame, the frequency and time of all precision atomic clocks are completely independent of their position and velocity. Hence, the absolute time as inherently measured by all precision atomic clocks, is completely independent of their position or velocity. The relativistic time is arbitrarily defined in SR such that the speed of light will always be measured to be isotropic in an IRF when the clocks are set to the relativistic time for that IRF. The relativistic time must change with change in position or velocity of the clocks in order to ensure isotropy of light speed in all IRF when measured with such clocks. Whereas in thought experiments of SR the clocks are assumed to automatically get adjusted to the relativistic time, real physical clocks can only be set to relativistic time through the process of e-synchronization.

Under the assumed isotropy of light speed in ECI frame, relativistic time gets distributed to the global Timing Labs by e-synchronous time transfer through satellite communication, whereas the aim was to distribute an absolute time. The absolute synchronization mismatch between two e-synchronized clocks is given by the relation (D.U) $/ \mathrm{c}^{2}$, where $\mathbf{D}$ is the separation distance (vector) between the two clocks and $\mathbf{U}$ is the absolute velocity vector (unknown) of the earth. This absolute synchronization offset between the master clocks at two distant timing Labs can be physically measured with an appropriate portable clock and such measurements can in fact be used to determine the unknown absolute velocity $\mathbf{U}$ of earth (Sandhu, 2012). After establishing the absolute velocity vector $\mathbf{U}$ of earth in the absolute or universal reference frame, we can easily account for the light speed anisotropy in ECI reference frame.

Consultative Committee for Time and Frequency (CCTF-19, 2012) in its $19^{\text {th }}$ meeting in September 2012, has recommended that all concerned agencies must actively encourage and support research aimed at significantly improving time and frequency transfer over intercontinental distances. For this it is absolutely necessary to,

$>$ Clearly distinguish between the notions of relativistic and absolute time.

$>$ Measure the absolute velocity of earth in the universal reference frame.

$>$ Account for the anisotropic speed of light in ECI reference frame. 
Thereafter, it will be possible to transfer the absolute time from highly stable and accurate primary frequency standards to the master clocks of global Timing Labs through satellite communication. With that, it should be possible to achieve absolute synchronization in space clocks and maintain absolute time in deep space flights.

\section{References}

BIPM (2012). Report on the activity and management of the international bureau of weights and measures supplement: time department. Retrieved from http://www.bipm.org/utils/common/pdf/DIR2012/time2012.pdf

Brunetti, G. (2011). Neutrino velocity measurement with the OPERA experiment in the CNGS beam. (Doctoral thesis, Alma Mater Studiorum, Bologna, Italy). Retrieved from http://operaweb.lngs.infn.it:2080/Opera/ptb/theses/theses/Brunetti-Giulia_phdthesis.pdf

CCTF-19. (2012). Consultative committee for time and frequency report of the 19th meeting (13-14). Retrieved from http://www.bipm.org/utils/common/pdf/CCTF19.pdf

Einstein, A. (1905). On the electrodynamics of moving bodies. Annalen der Physik, 17, 891. Retrieved from http://www.fourmilab.ch/etexts/einstein/specrel/www/

Einstein, A. (1916). Relativity: The special and general theory. New York: bartleby.com. Retrieved from http://www.bartleby.com/173/

OPERA Collaboration (T. Adam, et al.) (2011). Measurement of the neutrino velocity with the OPERA detector in the CNGS beam arXiv:1109.4897v4 [hep-ex]

Sandhu, G. S. (2009). Fundamental nature of matter and fields. iUniverse Inc. Bloomington, IN, 33. Retrieved from

http://bookstore.iuniverse.com/Products/SKU-000127260/Fundamental-Nature-of-Matter-and-Fields.aspx

Sandhu, G. S. (2010). Proposed experiment for detection of absolute motion. Physics Essays, 23(3), 442-450. http://dx.doi.org/10.4006/1.3454849

Sandhu, G. S. (2011a). Demystification of the spacetime model of relativity. Physics Essays, 24(1), 85-94. http://dx.doi.org/10.4006/1.3543877

Sandhu, G. S. (2011b). Relativity: a pillar of modern physics or a stumbling block. Proc. of SPIE 8121, 812109. http://dx.doi.org/10.1117/12.904607

Sandhu, G. S. (2012). Detection of absolute motion through measurement of synchronization offsets. Applied Physics Research, 4(4), 73-82. http://dx.doi.org/10.5539/apr.v4n4p73

Weiss, M., \& Thomas, C. (1994). NIST report-Implementation of a standard format for GPS common view data. Retrieved from http://www.dtic.mil/cgi-bin/GetTRDoc?AD=ADA518492

\section{Copyrights}

Copyright for this article is retained by the author(s), with first publication rights granted to the journal.

This is an open-access article distributed under the terms and conditions of the Creative Commons Attribution license (http://creativecommons.org/licenses/by/3.0/). 